\section{European study of fertility in Crohn's disease}

SIR,-We would like to thank Dr Purrmann and his colleagues (Gut 1986; 27: 1516) for their comments. They have failed to grasp the purpose of the study, however, which was to determine the overall effect of Crohn's disease on conception and pregnancy. To simply limit the study to the 182 married pairs would have failed to take account of national differences towards marriage and conception outside marriage, and also the effects of Crohn's disease on marriage itself. We rather chose to analyse all the cases, those who had been married at some time and those were were married at the time of the study. In this three level analysis the most significant differences in fertility were seen in the smaller group of 182 pairs, but significance was obtained for each group.

As to those women who had been advised against pregnancy or wished to avoid it less $(45 \%)$ took contraceptive precautions than did controls $(50 \%)$. It is quite possible that women with Crohn's disease are less sexually active than healthy controls but this is part of 'infertility' as was carefully defined in the paper, and is part of the disease.

This European report, which remains the first case controlled study, has clearly shown that women with Crohn's disease, despite less use of contraception, have less conceptions and less children than healthy controls. This is important practical information that can be given to concerned patients. Our next efforts in this field must be to identify the causes of this reduced fertility and where possible correct them.

Department of Therapeutics, Queen Medical Centre,

University Hospital of Nottingham,

Clifton Boulevard,

Nottingham.

\section{Books}

Infections of the gastrointestinal tract. Edited by $P$ D Manuel, J A Walker-Smith, A Tomkins. (Pp. 239; illustrated; £32.) Edinburgh: Churchill Livingstone, 1986.

As the number of new infections of the gastrointestinal tract increases, so do the published texts. This is certainly the most attractive publication so far, largely because it is presented in the double column format, extensively illustrated with line diagrams, black and white photographs and a multitude of Tables. The editors have assembled a small group of experts who in general have pro- duced excellent contributions, although some are less readable than others. The chapter on 'colitis' consists mainly of dense text without the relief of illustrations, which are so highly prevalent throughout the rest of the book.

Most topics are covered, although notable omissions are Aeromonas, Plesiomonas and Campylobacter pyloridis. The text leans gently towards the particular problems of infections in children, but this is hardly surprising from the interest and experience of the contributors. Nevertheless, this publication will be of great use to both adult and paediatric gastroenterologists alike, as it is easy to use as a reference source, contains practical guidance on management, and is extremely well referenced throughout.

M J G FARTHING

Liver failure Edited by Roger Williams. (Pp. 230; illustrated; £24) Edinburgh: Churchill Livingstone, 1986.

Liver failure is an increasingly common cause of death and in most patients the underlying cause is cirrhosis but in a few cases a previously healthy liver is suddenly the site of devastating damage usually by drugs or viruses. There has been a great deal of progress in the last 10 to 15 years in understanding the pathophysiological disturbances which lie behind the complex multifaceted clinical picture of liver failure and Dr Roger Williams has assembled an experienced team to review the field.

Hepatic encephalopathy is now thought to be caused by a disturbance in gamma-aminobutyric acid (GABA) metabolism and less importance is laid on ammonia metabolism and the false neurotransmitter hypothesis fashionable 10 years ago. E Anthony Jones presents this complex field lucidly. Cerebral oedema is a common and lethal development in hepatic failure and Ede and Williams emphasise the value of intracranial pressure monitoring and the use of intravenous mannitol. The marked cardiovascular and pulmonary changes in liver failure are the subject of a chapter well documented by the King's College Hospital experience. It has also recently been appreciated that changes in the microcirculation starve essential tissues of oxygen despite adequate systemic oxygen tension and cardiac output. The causes of renal failure which is such a common and lethal complication of liver failure are presented by Ring-Larsen. Hopefully these recent advances in understanding will lead to more effective treatment. Bacteriological infection is common and must be treated energetically. Cefotaxime has shown to be a more effective antibiotic than the previously favoured combination 
of ampicillin with tobramycin. There are contributions on the coagulopathy, antidotes to specific hepatotoxins such as paracetamol and the disturbances in amino acid metabolism. Oral and intravenous branch chain supplements have not lived up to early expectations. There is a fine chapter on the management of bleeding oesophageal varices based on the experience of the Barcelona liver unit but ascites gets little attention.

Finally the quest for the elusive artificial liver is brought up to date. Chang from McGill University who has done so much pioneering work in this field reviews the various systems of liver support and their experimental evaluation. The King's College Hospital experience of charcoal haemoperfusion, especially if this is started when the patient is still in grade three coma, is encouraging and the survival rate is much better than that of historical controls on series from other centres. They present evidence that the procedure may prevent the development of cerebral odema. Undoubtedly the best results of treatment do come from centres such as the Liver Failure Unit at King's where there is an experienced medical and nursing team.

Dr Roger Williams and his coauthors have produced a comprehensive and readable account of a complex subject and this book can be warmly recommended to all physicians dealing with patients with liver disease.

IAIN M MURRAY-LYON

Computed tomography of the gastrointestinal tract including the peritoneal cavity and mesentery Edited by M A Meyers. (Pp. 279; illustrated; DM248) Berlin: Springer-Verlag, 1986.

When computed tomography (CT) was first introduced, the solid intra-abdominal organs received almost undivided attention. The pancreas, liver, spleen, kidneys, and lymph nodes were extensively studied, the axial anatomy defined and the appearance of various pathologies were described in great detail. Hollow organ gastroenterology, the mesenteric cavities, the omentum and mesentery were considered only in passing, but quite shortly thereafter the diagnosis of mesenteric cysts and pseudocysts and of peritoneal abscesses gained prominence.

Later CT was applied to demonstrating the fascial planes particularly of the retroperitoneum, the various peritoneal cavities and recesses and to the mesentery and omentum. Now the oesophagus, stomach, small bowel, and colon also receive considerable attention. In some centres even to the extent of recommending CT as the first line of investigation in diverticular disease. The arguments in favour are by no means as fanciful as may initially appear. The complications of diverticular disease, bowel wall thickening, pericolic inflammation, abscess and fistula are well demonstrated. Furthermore CT is considerably more acceptable and less 'invasive' than a barium enema particularly in the elderly and is also more informative. A similar case can be made for diagnosing appendicitis or the complications of inflammatory bowel disease, not to mention tumours such as lymphoma and leiomyoma.

The expanded view of $\mathrm{CT}$ in gastrointestinal diagnosis is expertly presented in this book by world authorities who in the past have contributed greatly to our understanding of conventional radiology. This well written account, excellently illustrated and well presented can be recommended without reservation not only to radiologists but equally to surgeons and physicians interested in the gut. Sadly most NHS patients are still denied this facility and one wonders when District General Hospitals, let alone Teaching Hospitals, in this country will have immediate access to computed tomography and in particular for gastrointestinal tract disease.

L KREEL

\section{Books received}

Motility: a forgotten factor in gastrointestinal disorders?

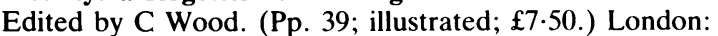
Royal Society of Medicine, 1985.

Tropical disease research 7 th prog report by World Health Organisation. (Pp. 14/10; illustrated; Sw fr 45.) Geneva: WHO, 1985.

Echographie du foie et des voies biliaares Edited by Andre Charbonnier. (Pp. 144; illustrated; FF96.) Paris: Masson, 1985.

\section{News}

International Conference on Endotoxins II

This conference will be held in Amsterdam from 21-23 May, 1987. Further details from: Scientific Secretary, ICEA II, Dr A Sturk, Academic Medical Center, F4-209, 1105 AZ Amsterdam, The Netherlands. Tel: (20) 5665976.

International Symposium on Immunology of the GI Tract and Liver

This symposium will be held at Laromme Hotel, Jerusalem, Israel from 22-27 March, 1987. Further details from: Symposium Secretariat International Ltd, 12 Shlomzion Hamalka St, Jerusalem, 94146, Israel. 